Nephrolithen

\section{Mittelmeerdiät beugt Nierensteinen vor}

— Eine mediterrane Diät hilft nicht nur, den Blutdruck zu senken, sondern wirkt sich auch positiv auf die Entstehung von Nierensteinen aus. Ein US-amerikanisches Forscherteam wollte nun herausfinden, welche Bestandteile der DASH-Diät (Dietary Approaches to Stop Hypertension), die nuss-, obst- und gemüsereich, aber arm an Tierprotein und gesüßten Getränken ist, einen lithoprotektiven Effekt haben. Dazu wurden 3.426 Personen nach ihren Ernährungsgewohnheiten befragt, zudem wurde der 24-Stunden-Sammelharn der Probanden auf lithogene Faktoren untersucht.

Beim Vergleich der obersten DASH-Quantile, also der Teilnehmer, die sich mutmaßlich am gesündesten ernährten, mit der untersten DASH-Quantile ergab sich folgendes: In der obersten Quantile war der Oxalatanteil im Urin bis zu $18 \%$ höher $(p<0,03)$, der Citratanteil bis zu 16\% höher $(p<0,01)$ und es wurde $32 \%$ mehr Gesamturin gebildet.

Demnach könnte eine Mittelmeerdiät durch eine erhöhte Citratkonzentration und Harnmenge das Harnsteinrisiko senken. Die Forscher vermuten zudem, dass bisher unbekannte Bestandteile aus Obst, Gemüse, Nüssen und Milchprodukten zur lithoprotektiven Wirkung beitragen.

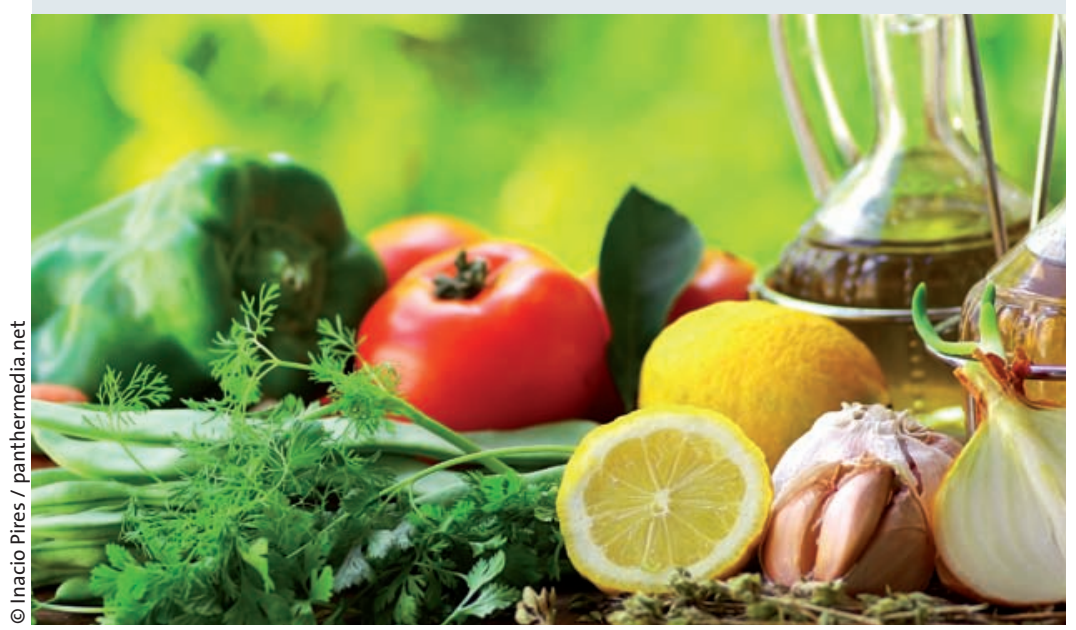

Unter anderem der hohe Citratanteil einer mediterranen Diät hemmt die Entstehung von Nierensteinen.

\section{Entscheidung des G-BA}

\section{Künstliche Befruchtung auch für Paare mit HIV}

— Paare mit HIV können die künstliche Befruchtung in Zukunft als Leistung der Gesetzlichen Krankenversicherung in Anspruch nehmen. Die bislang bestehende Ausschlussklausel hat der Gemeinsame Bundesausschusses (G-BA) im September aus der Richtlinie gestrichen. Bisher galt, dass beide Ehepartner zum Zeitpunkt einer künstlichen Befruchtung HIV-negativ sein mussten. Damit konnten HIV-infizierte Ehepartner diese Leistung nicht als Kassenleistung in Anspruch nehmen. Eine Ausschlussklausel für andere Erkrankungen gab es allerdings nicht.

Der Beratungsantrag wurde nach Angaben des G-BA von der Patientenvertretung vor allem damit begründet, dass HIV-positiven Versicherten die Leistungen schon wegen der Gleichbehandlung gegenüber Versicherten mit anderen Erkrankungen zur Verfügung stehen müssten. In der Begründung des Beschlusses legt der GBA dar, dass schwerwiegende Erkrankungen bei einem oder bei beiden Partnern ohnehin eine medizinische Bewertung des individuellen Risikos einer Schwangerschaft oder deren Herbeiführung durch Maßnahmen der künstlichen Befruchtung erfordern. „Ein Sonderstatus der HIV-Infektion lässt sich in diesem Zusammenhang medizinisch nicht begründen“, sagte Dr. Harald Deisler, unparteiisches Mitglied des G-BA und Vorsitzender des Unterausschusses Methodenbewertung. Der Beschluss des G-BA muss nun dem Bundesgesundheitsministerium zur Prüfung vorgelegt werden. Wird er dort nicht beanstandet, tritt er nach der Bekanntmachung im Bundesanzeiger in Kraft.

\title{
Prostatakarzinom
}

\section{Nanopartikel-PSA-Test erkennt Rezidive}

- Der konventionelle Test auf prostataspezifisches Antigen (PSA) zeigt bei bis zu $40 \%$ der Prostatektomiepatienten Prostatakarzinomrezidive nicht an. Der Test ist für die geringfügigen PSA-Schwankungen, die hier eine Rolle spielen, nicht sensitiv genug. Ein Team von US-amerikanischen Wissenschaftlern entwickelte nun auf Basis der Nanotechnologie einen Nanosphere-PSA-
Test (nPSA-Test), der 300-mal empfindlicher ist als der konventionelle PSA-Test. Damit untersuchten sie retrospektiv eingefrorene Seren von 188 Männern, die vor zehn Jahren eine radikale Prostatektomie durchgemacht hatten. Mit dem konventionellen Test war in diesen Seren kein PSA nachweisbar. Mit dem nPSA-Test wurden konstant niedrige PSA-Spiegel $(5,8$ pg/ml) über mindes- tens zehn Jahre nachgewiesen, was als Hinweis auf eine Heilung des Prostatakarzinoms gewertet wurde. Das nachgewiesene PSA stammt wahrscheinlich von den periurethralen Drüsen. In einigen Seren fanden sich aber höhere $(10,1 \mathrm{ng} / \mathrm{ml}$; $p=0,016)$ und ansteigende PSA-Werte. DiesePatienten hattenein Prostatakarzinomrezidiv.

km 